\title{
Influencia de la climatología, según la clasificación de Koppën e índice de Lang, sobre la tasa bruta de mortalidad asociada al cáncer de próstata en el área geográfica de España.
}

\author{
D. Santos Arrontes, M. Fernández Arjona, M.P. Valer López-Fando*, I. Cortés Aránguez. \\ Servicio de Urología. Hospital Santa Bárbara. Puertollano. Ciudad Real. \\ *Servicio de Gastroenterología. Hospital de Fuenlabrada. Madrid.
}

Actas Urol Esp 2005; (9): 828-833

\section{RESUMEN}

INFLUENCIA DE LA CLIMATOLOGÍA, SEGÚN LA CLASIFICACIÓN DE KOPPËN E ÍNDICE DE LANG, SOBRE LA TASA BRUTA DE MORTALIDAD ASOCIADA AL CÁNCER DE PRÓSTATA EN EL ÁREA GEOGRÁFICA DE ESPAÑA.

Objetivo: Evaluar si los factores climatológicos de temperaturas y precipitaciones influyen sobre la mortalidad asociada al cáncer de próstata a lo largo de un periodo de cinco años.

Material y Métodos: Se evalúan las tendencias de mortalidad asociada al cáncer de próstata en el periodo comprendido entre el 1 de enero de 1998 y el 31 de diciembre de 2002, en el área geográfica de España. Los datos demográficos y de mortalidad se obtuvieron del Instituto Nacional de Estadística y los datos climatológicos de temperaturas y precipitaciones se obtuvieron del Instituto Nacional de Meteorología aplicándose los valores registrados en el año 2002.

A partir de los datos meteorológicos se clasifican las diferentes provincias de acuerdo a la clasificación climática de Koppën, que los define a partir de los valores medios anuales y mensuales de temperatura y precipitación.

Las tasas de mortalidad serán expresadas por 100.000 habitantes y año (100.000 varones año en el caso del cáncer de próstata) aplicándose para su estudio el test de Kruskall-Wallis para "n" muestras independientes mediante el software SPSS v12.0 para Windows.

Resultados: Se obtienen tres áreas climáticas fundamentales, un área con clima tipo B que representa el $13 \%$ de la población total, un clima Csa y otro Csb que representan el 67 y el $20 \%$ respectivamente. La mortalidad hallada para el cáncer de próstata es mayor de forma estadísticamente significativa en el área climática Csb ( $\mathrm{p}=0,007)$, cuando se compara con las otras dos áreas climáticas.

Conclusión: La mortalidad asociada al cáncer de próstata es significativamente superior en las regiones con menor exposición solar. No obstante se requieren estudios prospectivos randomizados que confirmen estos hallazgos.

Palabras Clave: Neoplasias prostáticas. Epidemiología. Luz solar.

\section{ABSTRACT}

INFLUENCE OF CLIMATE, ACCORDING TO KOPPËN CLASSIFICATION AND LANG`S INDEX, ON GROSS RATE MORTALITY ASSOCIATED WITH PROSTATE CANCER IN THE GEOGRAPHICAL AREA OF SPAIN

Objective: To evaluate if the climatic factors of temperature and rainfall influence mortality associated with prostate cancer for a period of five years.

Material and methods: The mortality trends associated with prostate cancer will be evaluated in the period ranging from $1^{\text {st }}$ january 1998 to $31^{\text {st }}$ december 2002, in the geographical area of Spain. The demographic and mortality data were obtained from the National Institute of Statistics and the climatologically data on temperature and rainfall were obtained from the National Meteorology Institute applying the values registered in 2002 .

Based on the meteorological data, the different provinces are classified in accordance with Koppën climate classification and climatic index of Lang that defines them from the mean annual and monthly temperature and rainfall data.

Mortality rates will be expressed by 100.000 men year, applying for their study the Kruskall-Wallis test for "n" independent samples through the SPSS v12.0 Windows software.

Results: Three main climatic areas are obtained, one area with type B climate, which accounts for $13 \%$ of the total population, a Csa climate, and a Csb, accounting for 67 and 20\% respectively. The mortality found is higher in the Csb type for prostate cancer $(\mathrm{p}=0.007)$, as compared to the other two climatic areas.

Conclusion: Mortality associated with prostate cancer is significantly higher in the regions with a lower sun exposure. However, randomized prospective studies confirming these findings are warranted.

Keywords: Prostate neoplasm. Epidemiology. Sunlight. 
$\mathrm{L}^{\mathrm{a}}$ a patología oncológica constituye la segunda causa de mortalidad en los países desarrollados, superada únicamente por las enfermedades cardiovasculares. En concreto en España el 26,5\% de la mortalidad global es debida a enfermedad tumoral $(97,784$ fallecidos en el año 2002). Esta alta tasa de mortalidad asociada a patología tumoral está en relación, entre otros muchos factores, con el consumo de tabaco, que en España ha experimentado un repunte en los últimos cinco años. Así, mientras en 1997 un 33,1\% de los españoles reconocía fumar de forma diaria, en el año 2001 esta proporción se elevó hasta el $34,4 \%$ según datos de la Encuesta Nacional de Salud elaborada en $2003^{1}$.

No obstante, la mortalidad oncológica no tiene una distribución uniforme en todo el territorio nacional. De esta forma, en el año 2002 en Cantabria fallecieron 14,2/100.000 habitantes por neoplasia vesical, mientras que en Andalucía fueron $9,37^{1}$.

Uno de los factores que puede estar implicado en estas diferencias de mortalidad, teniendo en cuenta que se trata de un marco con una tradición histórica común, es la diversidad climatológica. Si tenemos en cuenta, que en ciertos procesos oncológicos se ha demostrado una relación directa con los niveles de exposición solar, por ejemplo, en el melanoma ${ }^{2}$, o indirecta, por ejemplo en el cáncer de colon (por mediación de un incremento de los niveles de vitamina D), es muy posible que la climatología desempeñe algún papel $^{3}$.

El objetivo de este estudio es evaluar si los factores climatológicos influyen sobre la mortalidad asociada al cáncer de próstata a lo largo de un periodo de cinco años.

\section{MATERIAL Y MÉTODOS}

En este estudio de tipo ecológico se evaluarán las tendencias de mortalidad asociada a los tumores de próstata en el periodo comprendido entre el 1 de enero de 1998 y el 31 de diciembre de 2002, en el área geográfica de España (17 Comunidades Autónomas -CA- y dos Ciudades Autónomas -Ceuta y Mellilla-, 43.000.000 habitantes).

Los datos demográficos y de mortalidad se obtuvieron del Instituto Nacional de Estadística $(\text { INE })^{1}$ ) y los datos climatológicos de temperaturas y precipitaciones se obtuvieron del Instituto Nacional de Meteorología (INM) ${ }^{4}$.

A partir de los datos meteorológicos se clasifican las diferentes provincias constituyentes de las CA de acuerdo a la clasificación bioclimática de Koppën ${ }^{5}$. En ella, los climas son definidos por los valores medios anuales y mensuales de temperatura y precipitación, así como por el tipo de vegetación predominante. Con estos criterios quedan definidos cinco grandes grupos climáticos (Tabla 1).

Para la asignación de los diferentes climas en cada región geográfica se utilizarán los datos meteorológicos correspondientes al año 2002, representándose la temperatura en grados centígrados y las precipitaciones en mililitros por metro cuadrado. Las tasas de mortalidad serán expresadas por 100.000 varones y año.

Además se evaluará la mortalidad en función de una serie de variables climáticas (temperatura media anual, temperatura máxima, temperatura mínima, precipitaciones totales anuales, humedad relativa media anual, número de días despejados, cubiertos y nubosos, número de días con temperaturas por debajo de $0^{\circ}$ y por encima de $25^{\circ}$ y número de horas de sol al año). Los valores de temperatura se registrarán en grados centígrados $\left({ }^{\circ} \mathrm{C}\right)$, los de precipitaciones en mililitros por metro cuadrado $\left(\mathrm{ml} / \mathrm{m}^{2}\right)$ y la humedad relativa en tanto por ciento.

Nuevamente, se clasificarán las diferentes provincias mediante el índice climático de Lang (definido como el cociente entre las precipitaciones anuales y la temperatura media anual). Se considerarán zonas áridas aquellas cuyo cociente sea inferior a $25 \mathrm{ml} / \mathrm{m}^{2} /{ }^{\circ} \mathrm{C}$, zonas semiáridas aquellas cuyo cociente se encuentre entre 25 y 40 $\mathrm{ml} / \mathrm{m}^{2} /{ }^{\circ} \mathrm{C}$, zonas húmedas aquellas que se encuentren entre 40 y $60 \mathrm{ml} / \mathrm{m}^{2} /{ }^{\circ} \mathrm{C}$, y muy húmedas cuando sea superior a $60 \mathrm{ml} / \mathrm{m}^{2} /{ }^{\circ} \mathrm{C}$.

Se determinarán las diferencias de cada una de las variables a estudio para cada región climática (árida, semiárida, húmeda y muy húmeda) de forma global mediante el test ANOVA, así como un análisis comparativo de los diferentes grupos con el área climática muy húmeda mediante el test de Dunnett. Se realizará un análisis multivariante mediante un modelo de re- 
Tabla 1

A Clima tropical Iluvioso. Todos los meses la temperatura media es superior a $18^{\circ} \mathrm{C}$. No existe estación invernal y las lluvias son abundantes.

B Climas secos. La evaporación es superior a la precipitación. No hay excedente hídrico. Se subdividen a su vez en áridos (BW) y semiáridos (BS), y cada uno puede diferenciarse aún más añadiéndole un tercer código, $\mathrm{h}$ para cálido y $\mathrm{k}$ para frío.

C Climas templados y húmedos. $\mathrm{El}$ mes más frío tiene una temperatura media comprendida entre $18^{\circ} \mathrm{C} \mathrm{y}$ $3^{\circ} \mathrm{C}$, y la media del mes más cálido supera los $10^{\circ} \mathrm{C}$.

D Climas templados de invierno frío. La temperatura media del mes más frío es inferior a $-3^{\circ} \mathrm{C}$ y la del mes más cálido está por encima de $10^{\circ} \mathrm{C}$.

E Climas polares. No tienen estación cálida y el promedio mensual de las temperaturas es siempre inferior a $10^{\circ} \mathrm{C}$. Cuando el mes más cálido oscila entre 0 y $10^{\circ} \mathrm{C}$ de temperatura media Köppen diferencia el grupo ET (Clima de tundra) y en el caso de que ningún mes supere los $0^{\circ} \mathrm{C}$ de temperatura media el grupo EF (Clima de hielo permanente). Son convencionalmente separados en tundra (ET) e Glacial (EF).

f Lluvioso todo el año, ausencia de periodo seco.

s Estación seca en verano.

w Estación seca en invierno.

m Precipitación de tipo monzónico.

a Temperatura media del mes más cálido superior a $22^{\circ} \mathrm{C}$.

b Temperatura media del mes más cálido inferior a $22^{\circ}$, pero con temperaturas medias de al menos cuatro meses superiores a $10^{\circ} \mathrm{C}$.

c Menos de cuatro meses con temperatura media superior a $10^{\circ} \mathrm{C}$.

d El mes más frío está por debajo de $-38^{\circ} \mathrm{C}$.

h Temperatura media anual superior a $18^{\circ} \mathrm{C}$.

k Temperatura media anual inferior a $18^{\circ} \mathrm{C}$.

Clasificación climatológica de Koppën. Los cinco grupos climáticos fundamentales serían los climas A, B, C, D y E, siendo el resto de variables accesorias para la definición de un clima. Se ha de incluir una característica de cada grupo, de esta forma ejemplos de definición climatológica serían: Afa, Bsh o Bwh.

gresión múltiple para cada variable en relación con las tasas de mortalidad. Además de ello se valorarán las diferentes tasas de mortalidad entre las distintas áreas climáticas mediante el test de Kruskall-Wallis para " $n$ " muestras independientes tanto para la región climática de Koppën como para el Índice de Lang. Todos los cálculos se realizarán mediante el software
SPSS v12.0 para Windows. Se considerará significativa una verosimilitud de la hipótesis nula inferior a 0,05 .

\section{RESULTADOS}

El tipo climático de Koppën para cada una de las provincias españolas, basadas en los valores de temperaturas y precipitaciones medias mensuales y anuales registradas en las diferentes estaciones meteorológicas repartidas por todo el territorio nacional, quedan representadas en la Figura 1.

Respecto a la clasificación de Koppën encontramos que todas aquellas localizaciones con precipitaciones inferiores a $250 \mathrm{ml} / \mathrm{m}^{2}$ al año (Almería y Las Palmas) corresponderían a climas tipo Bwh. Aquellas estaciones con precipitaciones inferiores a $300 \mathrm{ml} / \mathrm{m}^{2}$ y temperatura media anual superior a $15,5^{\circ}$ corresponderían a climas Bsh (Alicante y Murcia). Además también cumplen criterios de aridez Teruel, Zaragoza y Castellón (Bsh), esto es precipitaciones escasas de predominio estival (mayor evaporación), con temperatura media anual superior a $12^{\circ}$.

$\mathrm{El}$ resto de CA se corresponden a un clima tipo C (mes más frío superior a $0^{\circ}$ y menor de $18^{\circ}$, y con mes más cálido con temperatura superior a $10^{\circ}$ ), con una estación seca estival (Cs). A su vez se dividen en Csa y Csb (Coruña, Lugo, Pontevedra,

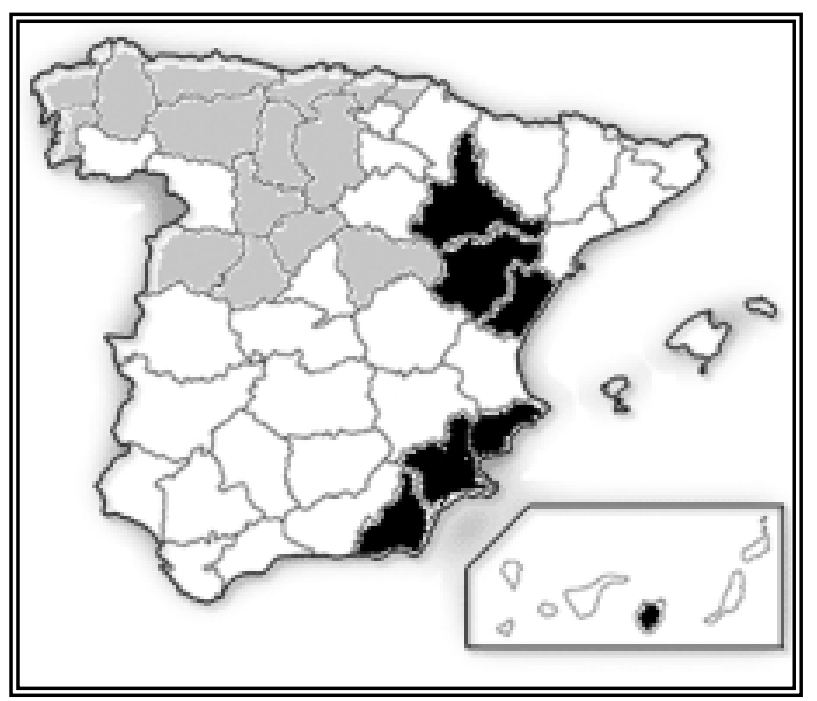

Figura 1. Distribución climatológica de España según la clasificación de Koppën, para el año 2002.

Color negro: Territorios con clima tipo $B$.

Color gris: Territorios con clima tipo Csb.

Color blanco: Territorios con clima tipo Csa 
Vizcaya, Guipúzcoa, Asturias, Cantabria, Castilla León - excepto Zamora y Soria- y Guadalajara).

Las poblaciones del área climática B en el año 2002, representa el $13 \%$ de la población total. las áreas geográficas de los climas Csa y Csb representan el 67 y el $20 \%$ respectivamente.

Los datos de mortalidad asociada al cáncer de próstata en las diferentes áreas climáticas, quedan representadas en la Tabla 2 , siendo superior de forma estadísticamente significativa en el área climática Csb $(\mathrm{p}=0,007)$, cuando se compara con las otras dos áreas.

Las zonas climática de acuerdo al índice de Lang, presentan diferencias estadísticamente significativas $(\mathrm{p}<0,05)$ mediante el test de Dunnett para cada una de las variables estudiadas, excepto la temperatura media anual entre la zona húmeda y muy húmeda $(\mathrm{p}=0,26)$, y la temperatura mínima anual entre la zona semiárida y húmeda $(p=0,28)$. Hay que resaltar que cuanto mayor sea el índice de Lang menor será la temperatura media anual, la temperatura máxima anual, el número de horas de sol al año, el número de días despejados, el número de días con temperaturas mayores de $25^{\circ}$ y el número de días con temperaturas por debajo de $0^{\circ}$. Es decir, se trata de climas lluviosos de temperaturas relativamente regulares a lo largo de todo el año, lo que equivaldría al clima de Koppën tipo Csb (Fig. 2 y Tabla 3).

Tabla 2

\begin{tabular}{ccccc}
\hline Cáncer próstata & Zona B & Zona Csb & Zona Csa & TOTAL \\
\hline \multirow{2}{*}{1998} & 763 & 1418 & 3547 & \\
& 29,13 & 35,75 & 27,49 & 5728 \\
& & & & \\
1999 & 29,22 & 35,46 & 26,53 & 5645 \\
& & & & \\
2000 & 25,24 & 1459 & 3313 & \\
& & & 25,50 & 5456 \\
2001 & 28,49 & 35,54 & 25,75 & 5659 \\
& & & & \\
2002 & 733 & 1490 & 3455 & \\
& 25,33 & 37,37 & 25,31 & 5678 \\
\hline
\end{tabular}

Registro de tumores genitourinarios en las diferentes áreas climáticas (1). El primer valor corresponde al número absoluto de tumores en cada área climática, el segundo valor corresponde a la tasa bruta de mortalidad asociada a cada tumor por 100.000 varones y año.

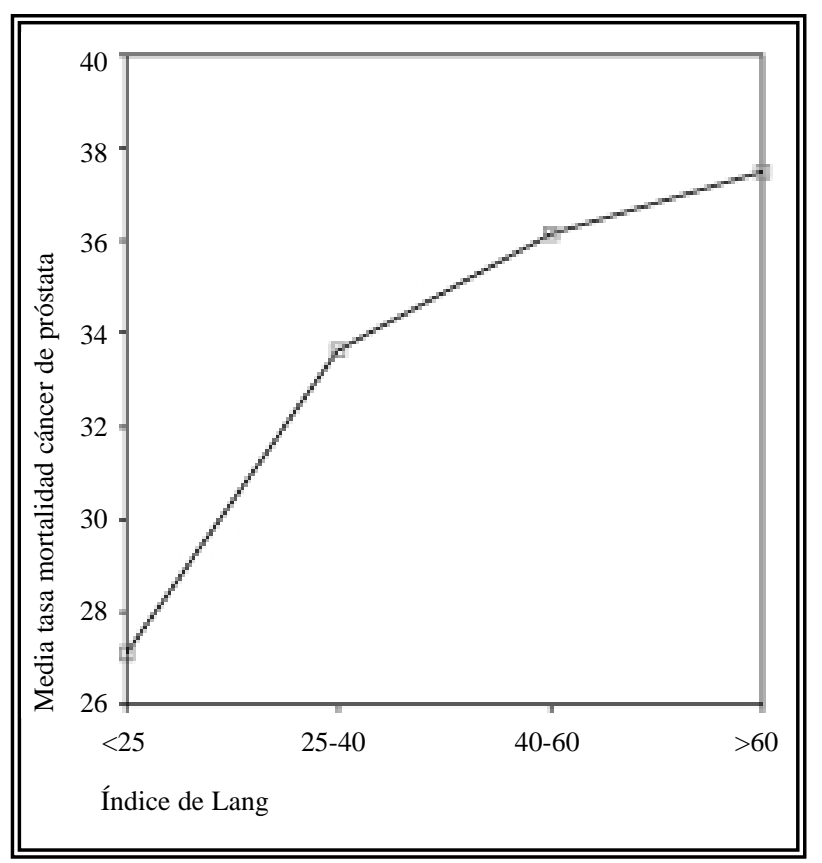

Figura 2. Representación gráfica de la mortalidad asociada a cáncer de próstata según el índice de Lang.

En el análisis multivariante para el estudio de la mortalidad asociada al cáncer de próstata, fueron significativas la temperatura media anual $(\mathrm{p}=0,01)$ y el número anual de horas de sol $(\mathrm{p}=0,022)$.

\section{DISCUSIÓN}

Los estudios ecológicos han puesto de manifiesto la posibilidad de la relación de la exposición a la luz solar con la reducción de la incidencia de cáncer de próstata. Estos estudios han sido confirmados por varios autores ${ }^{6,7,13}$. En el presente estudio nuevamente se confirma; las CA de la cornisa cantábrica son los que presentan un menor número de horas de exposición solar al año ${ }^{1}$ presentando la mayor tasa de mortalidad por cáncer de próstata. Por ejemplo, la tasa de mortalidad por cáncer de próstata en el área climática $\mathrm{B}$ es de 25,37 por cada 100.000 varones en el año 2002, mientras que en la zona Csb es de 37,37 .

Hay que tener en cuenta que como en todos los estudios ecológicos, los hallazgos encontrados deben ser confirmados por estudios prospectivos, debido a la existencia de importantes factores de confusión como por ejemplo la alimentación. Numerosos alimentos han sido involucrados en la prevención del cáncer de próstata, como los 
Tabla 3

\begin{tabular}{lcccccc}
\hline & T media anual & T max anual & T min anual & Precipitaciones & Humedad relativa & No horas de sol \\
Zona árida & 17,82 & 37,52 & 0,63 & 290,68 & 63,61 & 2923,93 \\
Zona semiárida & 15,18 & 37,1 & $-4,13$ & 481,72 & 63,53 & 2718,05 \\
Zona húmeda & 13,14 & 35,66 & $-5,84$ & 636,44 & 66,49 & 2397,95 \\
Zona muy húmeda & 13,86 & 33,9 & $-2,78$ & 1275,86 & 75,6 & 1952,23 \\
Regresión múltiple & 0,01 & 0,792 & 0,73 & 0,52 & 0,461 & 0,022 \\
\hline & Días despejados & Días nubosos & Días cubiertos & Días t > 250 & Días To $<0$ & Mortalidad \\
Zona árida & 103,21 & 213,28 & 44,65 & 145,27 & 13,68 & 27,09 \\
Zona semiárida & 94,91 & 200,47 & 67,21 & 121,77 & 32,17 & 33,62 \\
Zona húmeda & 60,73 & 204,87 & 97,31 & 89,84 & 42,89 & 36,13 \\
Zona muy húmeda & 44,15 & 188,86 & 130,96 & 50,7 & 14,95 & 37,48 \\
Regresión múltiple & 0,296 & 0,654 & 0,277 & 0,141 & 0,314 & p< $0,001^{*}$ \\
\hline
\end{tabular}

Distribución de los factores climáticos a estudio en las áreas con diferente Índice de Lang. El valor asociado a cada variable en la regresión múltiple muestra su significatividad respecto a la mortalidad asociada al cáncer de próstata. *Calculado mediante el test de Kruskall-Wallis, los valores que aparecen reflejados para cada área son las medias de mortalidad entre las diferentes zonas climáticas y periodos.

licopenos del tomate ${ }^{8}$, la soja ${ }^{9}$, el té verde ${ }^{10}$ o el vino tinto ${ }^{11}$, siendo la producción, consumo y procesamiento de los mismos muy variable, sobre todo de los productos derivados de la uva. Sin embargo, no es posible disponer de registros de venta o consumo de los diferentes alimentos en las distintas provincias.

Otro factor de confusión a tener en cuenta son los factores genéticos intrínsecos a los diferentes grupos de población de la Península Ibérica, así como los derivados de la inmigración. Esta última no se reparte igual en todas las provincias (siendo mayor en los principales grupos urbanos) pudiéndose modificar las tasas de mortalidad. Por ejemplo, los individuos de raza negra tienen una mayor predisposición a desarrollar cáncer de próstata $^{12}$.

Además existen varias limitaciones de relativa importancia. Por un lado la ausencia de registros oficiales de incidencia de cánceres a nivel nacional, de tal forma que todos los datos de mortalidad proceden de los certificados de defunción, siendo posible que en pacientes pluripatológicos se omitan enfermedades concomitantes que no sean la causa fundamental de la defunción. Por otro, el estudio está realizado sobre tasas brutas de mortalidad. Sería recomendable realizar nuevamente el estudio basándose en datos de incidencia, lo cual es bastante complicado, pues al no tratarse de una enfermedad de declaración obligatoria, las incidencias calculadas a partir de modelos matemáticos son en el mejor de los casos provisionales. Otra posibilidad, sería elaborarlo a partir de mortalidad cáncer específica ajustada por edad, que constituiría el siguiente paso lógico para confirmar estos hallazgos.

\section{CONCLUSIÓN}

La mortalidad asociada al cáncer de próstata es significativamente superior en las regiones con menor exposición solar. Dentro de las distintas variables a estudio la temperatura media anual aparece como el factor de riesgo más relevante. No obstante, estos resultados hay que tomarlos con precaución y deben ser confirmados por estudios prospectivos. Por último sería recomendable ampliar este estudio a otros tipos de tumores (pulmón, mama...).

\section{REFERENCIAS}

1. Instituto Nacional de Estadística. http://www.ine.es. Última entrada 22/1/2005.

2. Autier P. Perspectives in melanoma prevention: the case of sunbeds. Eur J Cancer. 2004;40(16):2367-2376.

3. Chakrabarty S, Wang H, Canaff L, Hendy GN, Appelman H, Varany J. Calcium sensing receptor in human colon carcinoma: interaction with $\mathrm{Ca}(2+)$ and 1,25-dihydroxyvitamin D(3). Cancer Res 2005;15(65):493-498.

4. Instituto Nacional de Meteorología. http://www.inm.es. Última entrada 22/1/2005.

5. En Jansa Guardiola JM. Curso de Climatología. Editorial Instituto Nacional de Meteorología, 1983.

6. Freedman DM, Dosemeci M, McGlynn K. Sunlight and mortality from breast, ovarian, colon, prostate, and nonmelanoma skin cancer: a composite death certificate based case-control study. Occup Environ Med. 2002;59(4):257267. 
7. Grant WB. A multicountry ecologic study of risk and risk reduction factors for prostate cancer mortality. Eur Urol. 2004;45(3):271-279.

8. Campbell JK, Canene-Adams K, Lindshield BL, Boileau TW, Clinton SK, Erdman JW Jr. Tomato phytochemicals and prostate cancer risk. J Nutr. 2004;134(12 Suppl): 3486S-3492S.

9. Holzbeierlein JM, McIntosh J, Thrasher JB. The role of soy phytoestrogens in prostate cancer. Curr Opin Urol. 2005; 15(1): 17-22.

10. Adhami VM, Siddiqui IA, Ahmad N, Gupta S, Mukhtar H. Oral consumption of green tea polyphenols inhibits insulin-like growth factor-I-induced signaling in an autochthonous mouse model of prostate cancer. Cancer Res. 2004;64(23):8715-8722.

11. Schoonen WM, Salinas CA, Kiemeney LA, Standford JL. Alcohol consumption and risk of prostate cancer in middle-aged men. Int J Cancer. 2005;1-113(1):133-140.
12. Hsieh K, Albertsen PC. Populations at high risk for prostate cancer. Urol Clin North Am. 2003;30(4):669676.

13. John EM, Dreon DM, Koo J, Schwartz GG. Residential sunlight exposure is associated with a decreased risk of prostate cancer. J Steroid Biochem Mol Biol. 200;89-90(15):549-552.

Dr. D. Santos Arrontes

Servicio de Urología

Hospital Santa Bárbara

Malagón, s/n

13500 Puertollano (Ciudad Real)

(Trabajo recibido el 6 de mayo 2005) 